\title{
ON THE OBLIQUITY OF THE ECLIPTIC
}

\author{
K.Y. CHEN \\ University of Florida \\ Gainesville, Florida 32611-2055, U. S. A.
}

\section{Introduction}

This study was originally motivated from the discussion of the obliquity of the ecliptic, $\epsilon$, in the work of Needham (1959) and his collaborators, and the article, "An 8-th Century Meridian Line," by Beer $e t$ al. (1961). A search in the historical records yields values of $\epsilon$ in addition to the published values in the literatures.

The gnomon-shadow lengths of the sun at noons on the solstitial days as recorded in the Chinese Annals were collected. Each summer-winter pair was used to compute the latitude of the observing site and the value of $\epsilon$. The values of $\epsilon$, determined in the Orient and in the Occident, are used to show the variation of $\epsilon$ in the three thousand years of recorded history.

\section{The Observations}

The heights of the Chinese gnomon were given as 8 chi; except the 9-chi gnomon used for the first listed set of observations at Jiankang, and the 40-chi gnomons used in Beijing. The calculated values are not corrected for the effects of atmospheric refraction and local height from the sea level. They are listed in Table 1. Column 2 lists the places of observation, column 3 the calculated values of latitudes and obliquity, column 4 the altitude of the sun at noon of the summer solstice. The latitudes and longitudes in the last column were copied from the Gazetteers of the U. S. Office of Geography, except those of Gaocheng which were measured in the twentieth century for the gnomon of the Duke of Zhou ( $d 1036$ B.C.E.). This monumental stone gnomon was elected by the astronomer royal Nangong Shuo in 723 C.E.. In Table 1, the angles are given in degrees, and the latitudes and longitudes are measured to the north and to the east, respectively.

The land of the State of Linyi has been known to China since the Han dynasty. Its capital is said to be near the ancient city of Hue. The value of $0.57 \mathrm{chi}$, which is recorded in the Tang Annals for gnomon-shadow length as measured by Da Xiang and Yuan Tai in Linyi on the summer-solstice day, does not appear to be correct. Instead, the value of $0.91 \mathrm{chi}$, which was obtained after a miliatary campaign of Liu Song against Linyi in 433 C.E., is used here. The land of Tiele was known as the land of Xiongnu in the Han dynasty. The calculated latitude of the place is not as far north as that of Lake Baykal. On his journey to Samarkand, the Taoist Qui Chu-ji measured the gnomon shadow of the sun on the summer-solstice day along the shore of the Kerulen river. The longitude of the place of the observation is estimated to be about $112^{\circ} \mathrm{E}$ according to the event written in The Journey West by Li Zhi-chang.

The correlation between the calculated latitutes and the geographic latitutes of the modern cities is an indication of the accuracy of the calculated values, including $\epsilon$. Using the regression analysis, one finds that Laiitude calc $_{c}=1.055+0.961 \times$ Latitude $_{\text {geo }}$, with the correlation coefficient of 0.998 . Similarly, one obtains that Latitude $_{\text {calc }}=23.596+1.003 \times$ alt. $\odot$, with the correlation coeficient of 1.000 .

Other published values of $\epsilon$ are collected and listed in Table 2. Again, the latitudes and longitudes in the last column are from the Gazetteers, except that of the first entry, in which the values were derived from Artha-sastra (ref. Y. Ohashi's article in this Joint Discussion). 
TABLE 1. Chinese Records with Gnomon Measurements

\begin{tabular}{|c|c|c|c|c|c|}
\hline Year & Place & Latitude/Obliquity & Altitude $\odot$ & City & Lat./Long. \\
\hline ca 1100 B.C.E. & Yangcheng & $34.51 / 23.89$ & 79.38 & Gaocheng & $34.41 / 113.14$ \\
\hline 442 C.E. & & - & 79.38 & & \\
\hline 724 & & $34.15 / 23.68$ & 79.52 & & \\
\hline 822 & & $34.16 / 23.69$ & 79.53 & & \\
\hline 1024 & & $34.15 / 23.68$ & 79.53 & & \\
\hline 442 & Jiaozhou & - & 87.85 & Hanoi & $21.03 / 105.85$ \\
\hline 442 C.E. & Linyi & - & 83.51 & $\ldots$ & - \\
\hline ca 300 B.C.E. & Luoyang & $35.05 / 23.73$ & 78.69 & Luoyang & $34.68 / 112.47$ \\
\hline $115-120$ C.E. & & $34.51 / 23.89$ & 79.38 & & \\
\hline 508 & & - & 78.83 & & \\
\hline $581-584$ & & $34.24 / 23.76$ & 79.52 & & \\
\hline $596-597$ & & $33.94 / 23.69$ & 79.73 & & \\
\hline 544 & Jiankang & $31.90 / 23.55$ & 81.67 & Nanjing & $32.05 / 118.78$ \\
\hline 544 & & - & 81.66 & & \\
\hline \multirow[t]{10}{*}{$721-725$} & Linyi & $17.14 / 23.63$ & 85.93 & - & - \\
\hline & Jiaozhou & $21.21 / 23.57$ & 87.64 & Hanoi & $21.03 / 105.85$ \\
\hline & Langzhou & $29.14 / 23.64$ & 84.50 & Changde & $29.03 / 111.68$ \\
\hline & Caizhou & $33.24 / 23.74$ & 80.35 & Runan & $33.02 / 114.37$ \\
\hline & Xuzhou & $33.83 / 23.62$ & 79.80 & Fugou & $34.07 / 114.38$ \\
\hline & Bianzhou & $34.46 / 23.63$ & 79.17 & Kaifeng & $34.85 / 114.35$ \\
\hline & Huazhou & $34.75 / 23.65$ & 78.90 & Huaxian & $35.58 / 114.50$ \\
\hline & Weizhou & $39.63 / 23.65$ & 74.03 & Weixian & $39.80 / 114.40$ \\
\hline & Tiele & $51.01 / 23.70$ & 62.70 & - & - \\
\hline & Henanfu & $34.18 / 23.63$ & 79.45 & Luoyang & $34.68 / 112.47$ \\
\hline $1049-1052$ & Junyi & $34.60 / 23.48$ & 78.88 & Kaifeng & $34.85 / 114.35$ \\
\hline 1064 & & $34.60 / 23.50$ & 78.90 & & \\
\hline 1092 & & $34.60 / 23.50$ & 78.90 & & \\
\hline 1106 & & $34.55 / 23.51$ & 78.97 & & \\
\hline 1221 & Kerulen R. & & 65.47 & $\cdots$ & - \\
\hline 1277 & $\mathrm{Da} \mathrm{Du}$ & $39.86 / 23.54$ & 73.68 & Beijing & $39.93 / 116.40$ \\
\hline 1368 C.E. & Beijing & $39.85 / 23.54$ & 73.68 & & \\
\hline
\end{tabular}

TABLE 2. Other Oriental Records

\begin{tabular}{cclc}
\hline Year & Obliquity & Place & Lat./Long. \\
\hline ca 300 B.C.E. & 23.7 & India & $23.7 /-$ \\
230 B.C.E. & 23.86 & Alexandria & $31.20 / 29.90$ \\
830 C.E. & 23.56 & Baghdad & $33.30 / 44.60$ \\
832 & 23.55 & Damascus & $33.50 / 36.25$ \\
869 & 23.55 & Baghdad & $33.30 / 44.60$ \\
880 & 23.58 & ar Raqqah & $36.00 / 39.00$ \\
970 & 23.58 & Shiraz & $29.60 / 52.53$ \\
970 & 23.58 & Cairo & $30.05 / 31.25$ \\
987 & 23.58 & Baghdad & $33.30 / 44.60$ \\
994 & 23.54 & Shahr Rey & $35.58 / 51.42$ \\
1009 & 23.58 & al-Mugattam & $30.03 / 31.28$ \\
1259 & 23.50 & Maragha & $37.33 / 46.38$ \\
1424 C.E. & 23.52 & Santarkand & $39.40 / 66.58$ \\
\hline
\end{tabular}

\section{The Saeculum of Decline}

A 2-degree polynomial is used to express the secular variation of the values of $\epsilon$ as listed in Tables 1 and 2 , togather with the value of $23.439^{\circ}$ for the epoch 1976.0. Using the method of least squares, 
one find that $\epsilon=23.73757813-0.00014623 \times T-0.00000001 \times T^{2}$, where $\mathrm{T}$ is the number of years elapsed since the first year C.E. The quantity, defned as the unexplained variation/the number of degrees of freedom and called $\beta$ here, is equal to 0.00341321. If the values of Ptolemy and Copernicus, and the 27 occidental observed values (Wittmann, 1984) are used togather with the 38 oriental values and the 1976 value, one obtains the 2-degree polynomial with the use of the method of least squares $\epsilon=23.73989867-0.00015320 \times T$, which is a linear function representing the secular decline in the value of $\epsilon$ at the rate of $0.01532^{\circ}$ per century. The value of $\beta$ is 0.00235874 .

\section{References}

Beer, A., Ho, P-Y., Lu, G-D., Needham, J., Pulleyblank, E.G., and Thompson, G.I. (1961) An 8th-Century Meridian Line, Vista in Astronomy, 4, pp. 3-28.

Needham, J. (1959) Science and Civilisation in China. University Press, Cambridge.

Whittmann, A.D. (1984) On the Variation of the Obliquity of the Ecliptic, Astronomische Gesellschaft Mitteilungen, Nr. 62, pp. 201-204. 